\title{
IMPLEMENTATION OF FINITE VOLUME METHOD IN CREEPING FLOW AROUND A CIRCULAR CYLINDER
}

\author{
M. Y. Gokhale ${ }^{1}$, Mansoureh Sarbandi Farahani ${ }^{2}$ \\ ${ }^{1}$ Maharashtra Institute of Technology, Pune, Maharashtra, India \\ ${ }^{2}$ Department of Mathematics, S. P. Pune University, Pune, Maharashtra, India
}

\begin{abstract}
A Finite Volume Method have been performed in simulation of creeping flow around a circular cylinder contained between plates. By adopting the SIMPLE algorithm the governing equations are solved together with Papanastasious regularization. Apparent viscosity is calculated on each iteration by using forward difference operation. Yield surfaces are studied over the range of Oldroyd number $10 \leq \mathrm{Od} \leq 10^{4}$. The model results are found to be in good agreement with obtained results of the other method.
\end{abstract}

Keywords: Creeping flow, Finite Volume Method, Yielded and unyielded zone, Bingham number. - $* * *$

\section{INTRODUCTION}

Creeping flows characterized by very low the Reynolds number $(\operatorname{Re} \leq 1)$, which is dominated by viscosity and occur in many fluids such as honey, paste food and blood. In such fluids when the stress falls the yield stress, three rigid zones formed in the vicinity of the cylinder. The study of identifying the location and size of rigidzones has been of interest to researchers.

Over the range $0.01 \leq \mathrm{Bn} \leq 1000$, Mitsoulis [8] Extensive results on the location and size of unyielded regions, has reported the effect of the Bingham number on the size of various unyielded regions around the cylinder. Tokpavi et al. [13] adopted Papanastasious regularization with finite element methods to study flow around a circular cylinder, they have been identified and characterized the rigid zones over the wide range of Oldroyd number. Using the finite element method, Nirmalkar et al. [10] simulated the 2Dcreeping flow around square cylinder over a wide range of Bingham number as $1 \leq \mathrm{Bn} \leq 10^{5}$. They are identified three zones of unyielded regions in the vicinity of the cylinder, all zones expanded by increasing Bingham number. This study investigates 2D-creeping flow past a circular cylinder by adopting a Finite Volume Method, Semi Implicit Method for Pressure-Linked Equation (SIMPLE) is used for coupling the pressure and velocity. The governing equations are solved numerically together with the Papanastasiou model [11].

Yield surfaces are predicted over the range of Oldroyd number $1 \leq \mathrm{Od} \leq 10^{4}$. The objective of this work is implementing Finite Volume Method for Non-Newtonian flow problems as an alternative solver to the Finite Element Method and commercial software like COMSOL. The results are validated with numerical results of Tokpavi et al.[13].

\section{GOVERNING EQUATIONS}

The problem of 2D-creeping flow past a circular cylinder of diameter $\mathrm{d}$ is considered (Fig 1).

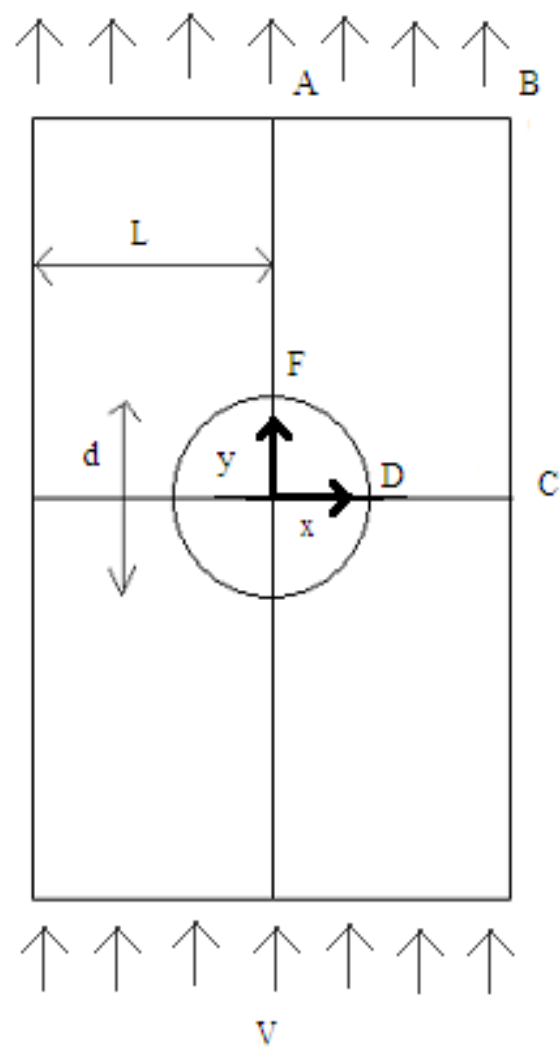

Fig -1: Flow field. Schematic representation of the problem

The length of the domain is $2 \mathrm{~L}$ and cylinder is situated halfway between two flat plates $2 \mathrm{~L}$ apart. Because of the creeping flow assumption ( $\operatorname{Re}=0)$ there is symmetry in both $\mathrm{x}$-direction and $\mathrm{y}$-direction in a Cartesian coordinate $\operatorname{system}(\mathrm{x}, \mathrm{y})$. 
For an incompressible fluid the conservation equations of mass and momentum in the condition of creeping flow can be written as:

$\nabla \cdot \bar{v}=0$

$-\nabla \mathrm{P}+\nabla \cdot \overline{\bar{\tau}}=0$

Where $\overline{\mathrm{v}}$ is the velocity vector, $\overline{\bar{\tau}}$ the extra-stress tensor, and $\mathrm{p}$ is the scalar pressure. The Bingham constitutive equation is:

$$
\begin{cases}\tau=\tau_{\mathrm{y}}+\mu \dot{\gamma} & |\tau|>\tau_{\mathrm{y}} \\ \dot{\gamma}=0 & |\tau| \leq \tau_{\mathrm{y}}\end{cases}
$$

Where $\tau$ is the shear stress, $\tau_{\mathrm{y}}$ the yield stress, $\mu$ the constant plastic viscosity and $\dot{\gamma}$ is the rate of strain tensor,

$$
\dot{\gamma}=\left(\nabla V+\nabla V^{T}\right)
$$

$V$ is the velocity. In order to avoid the discontinuity in viscoplastic model Papanastasiou [11] modified the Bingham model as:

$$
\overline{\bar{\tau}}=\left(\mu+\tau_{\mathrm{y}} \frac{1-\exp (-m \dot{\gamma})}{\dot{\gamma}}\right) \overline{\bar{\gamma}}
$$

Where $\mathrm{m}$ is the stress growth exponent, the magnitude of the rate of the deformation tensor and the deviator stress tensor are given by:

$$
|\dot{\gamma}|=\sqrt{\frac{1}{2} \operatorname{tr}\left(\dot{\gamma}^{2}\right)} \quad|\tau|=\sqrt{\frac{1}{2} \operatorname{tr}\left(\tau^{2}\right)}
$$

The Oldroyd number which is the ratio of the yield stress to viscous stress can be written as:

$$
B_{n}=\frac{\tau_{0} d}{\mu V}
$$

All variables are rendered dimensionless using $\mathrm{d}, \mathrm{V}$ and $\tau_{0}$ as scaling variables for length, velocity and stress component respectively.

\subsection{Boundary Conditions}

The flowing is very slow then, the inertia forces are negligible. Because of symmetry it is necessary to consider only one quadrant of the flow domain, as was done previously by Beris et al. [3] in the case of flow around a sphere.

Fig 1 shows the solution domain and boundary conditions. The boundary conditions are:

- Symmetry along $\operatorname{AF}\left(u=0, \frac{\partial v}{\partial x}=0\right)$

- Symmetry along $\operatorname{DC}\left(u=0, \frac{\partial v}{\partial y}=0\right)$

- On the boundary $\mathrm{AB}$ and $\mathrm{BC}(u=0, v=V)$

- No slip at the cylinder surface along $\operatorname{FD}(u=v=0)$

- The reference pressure is set to zero at point $\mathrm{C}$

\subsection{Unyielded and Yielded Zone}

Unyielded (rigid) zones refer to all areas in which inequality $\tau<\tau_{\mathrm{y}}$ satisfied, otherwise are called yielded zones and be considered as a fluid. The interface between unyielded and yielded zones is called yield surface $\left(\tau=\tau_{\mathrm{y}}\right)$. Three distinct rigid zones as shown in Fig2, with their corresponding yield surfaces are observed by Tokpavi et al. [13].

- A far-field Dynamic rigid zone (Zr1)

- Two static rigid zones with triangular shape connected to the cylinder $(\mathrm{Zr} 2)$

- Two dynamic zones on both sides of the cylinder equidistant from it $(\mathrm{Zr} 3)$

- The yield surface S1, boundary between $\mathrm{Zr} 1$ and fluid zone.

- The yield surface S2, boundary between Zr2 and fluid zone.

- The yield surface S3, boundary between Zr3 and fluid zone.

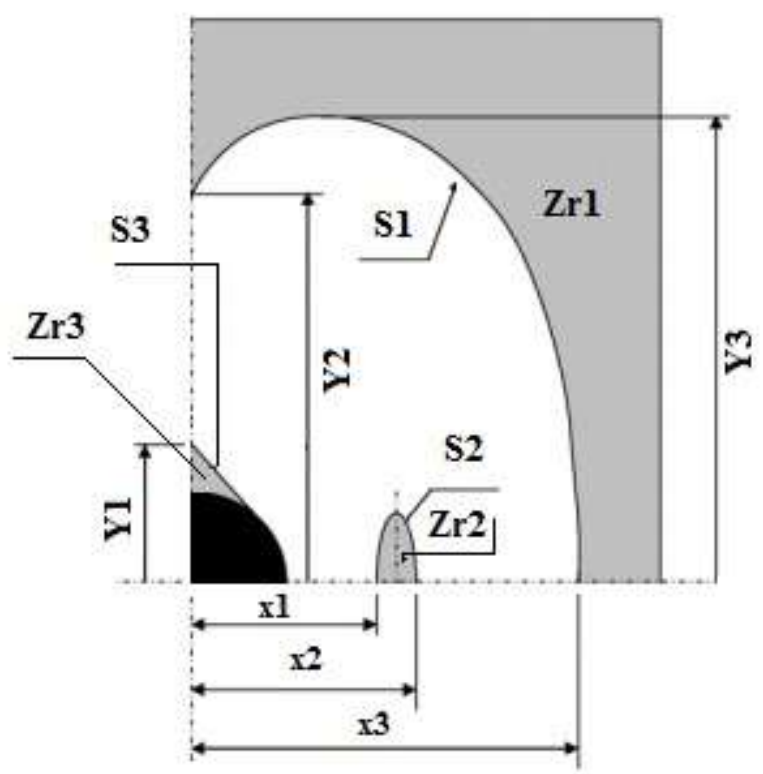

Fig-2: Definition of yield surfaces, rigid and fluid zones

\section{COMPUTATIONAL PROCEDURES}

The SIMPLE algorithm developed here in rectangular Cartesian coordinate system, the location of the depended variable shown in Fig 3. The pressure p, stored in the cell midpoint and the velocities $\mathrm{u}$ and $\mathrm{v}$ placed on the vertical and horizontal cell interface respectively. The constitutive equation must be solved together with the momentum equations. Using the center difference approximation, the $u$ momentum and v-momentum discretized on the point the index of (i,J), and (I,j) respectively (Fig 4$)$, the continuity equation and the constitutive equation discretized on the point the index of $(\mathrm{I}, \mathrm{J})$. 


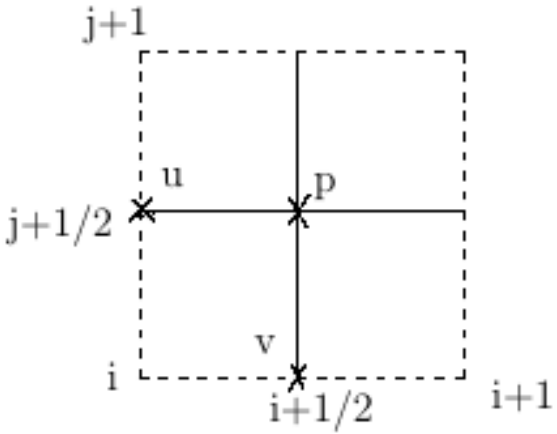

Fig-3: Control volume cell

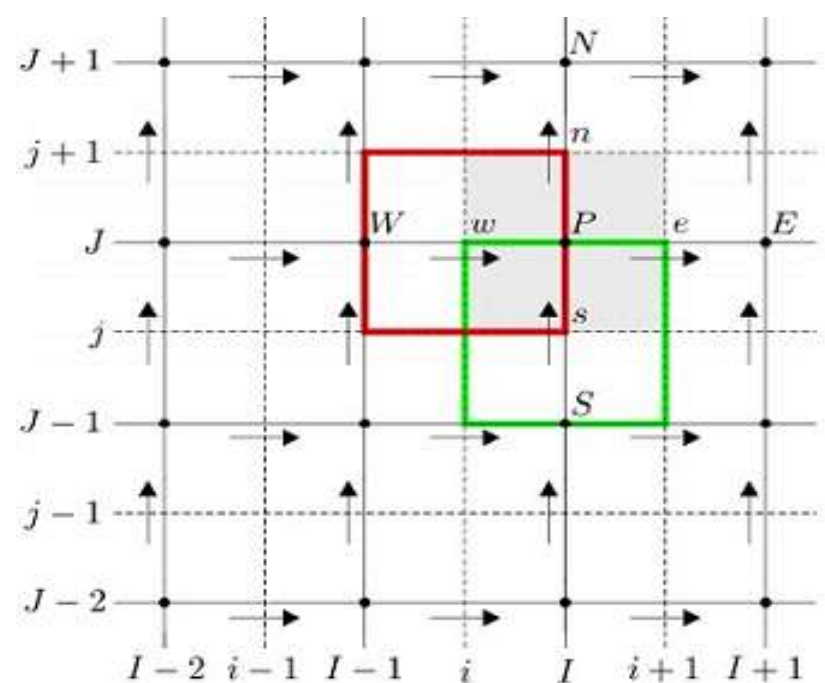

Fig-4: Staggered grid

\subsection{Solution Algorithm}

- Step1: Initialize the velocity and pressure

- Step 2: Evaluate the apparent viscosity by using Equation (5)

- Step 3: Set initial guess for $p^{*}, u^{*}, v^{*}$

- Step 4: Evaluate $u^{*}$ by solving discretized $\mathrm{u}-$-momentum equation

$a_{i, J} u_{i, J}^{*}=a_{i-1, J} u_{i-1, J}^{*}+a_{i+1, J} u_{i+1, J}^{*}+a_{i, J-1} u_{i, J-1}^{*}$

$+a_{i, J+1} u_{i, J+1}^{*}+S_{u} \Delta V-\frac{p_{I, J}-p_{I-1, J}}{\Delta y} \Delta V+$

$\left(1-\alpha_{u}\right) u_{i, J}^{n-1}$

- Step 5: Evaluate $v^{*}$ by solving $\mathrm{v}$-momentum equation approximated

$a_{I, j} v_{I, j}^{*}=a_{I, j-1} v_{I, j-1}^{*}+a_{I+1, j} v_{I+1, j}^{*}+a_{I-1, j} v_{I-1, j}^{*}$

$+a_{I-1, j} v_{I-1, j}^{*}+S_{v} \Delta V-\frac{p_{I, J}-p_{I, J-1}}{\Delta y} \Delta V+$

$\left(1-\alpha_{v}\right) v_{I, j}^{n-1}$

- Step 6: Evaluate $p$ 'by solving Poisson equation

$$
\begin{aligned}
a_{I, J} p_{I, J}^{\prime}= & a_{I+1, J} p_{I+1, J}^{\prime}+a_{I-1, J} p_{I-1, J}^{\prime}+a_{I, j+1} p_{I, j+1}^{\prime}+ \\
& a_{I, J-1} p_{I, J-1}^{\prime}+b_{I, J}^{\prime}
\end{aligned}
$$

- Step 7: Correct pressures and velocities using underrelaxation

$$
\begin{gathered}
p^{n}=p^{* n-1}+\alpha_{p} p^{n} \\
u_{i, J}^{\prime}=-\left(p_{I, J}^{\prime}-p_{I-1, J}^{\prime}\right) \frac{\Delta x_{i, J}}{a_{i, J}}, \quad v_{I, j}^{\prime}=-\left(p_{I, J}^{\prime}-p_{I, J-1}^{\prime}\right) \frac{\Delta x_{I, j}}{a_{I, j}} \\
u^{n}=\alpha_{u}\left(u^{* n}+u^{\prime n}\right)+\left(1-\alpha_{u}\right) u^{n-1} \\
v^{n}=\alpha_{v}\left(v^{* n}+v^{n}\right)+\left(1-\alpha_{v}\right) v^{n-1}
\end{gathered}
$$

- Step 8: Increase iteration step, if iteration step $<\max$ iteration, go to step 2

\section{RESULT AND DISCUSSION}

In this problem, a circular cylinder contained between two plates, the momentum equation and continuity equation are solved together with Papanastasiou equation [11]. The SIMPLE algorithm is used as a pressure correction method. Numerical computation is carried out on the impute values which correspond to the experiment conducted by Tokpavi et al. , the schematic diagram of the $2 \mathrm{D}$-creeping flow past a circular cylinder is shown in Fig 1 . The cylinder of diameter $\mathrm{d}$ is situated half-way between two plates, as inertia force is neglected, two plates of symmetry are considered.

Thus, the domain is reduced to the quarter of the flow field. The computational domain comprises $\mathrm{L} \times \mathrm{L}$, the ratio length by cylinder diameter is considered $\mathrm{L} / \mathrm{d}=5$. The symmetry boundaries are considered at the AF and DC. The velocity $\mathrm{u}$ and $\mathrm{v}$ at the $\mathrm{AB}$ and $\mathrm{BC}$ are specified as zero and $\mathrm{V}$ respectively. The no slip boundary is specified at the cylinder. Numerical simulation is performed by considering $\mathrm{nXp} \times \mathrm{nYp}, \mathrm{nXp}-1 \times \mathrm{nYp}$ and $\mathrm{nXp} \times \mathrm{nYp}-1$ cells to store the pressure, u-velocity and v-velocity values respectively. Fig 5 shows the computational grid, in the vicinity of the cylinder a finer mesh is used (grid size $\mathrm{h}=$ 0.005 in the vicinity of cylinder and $h=0.01$ for far from the cylinder, totally 1900 cells).

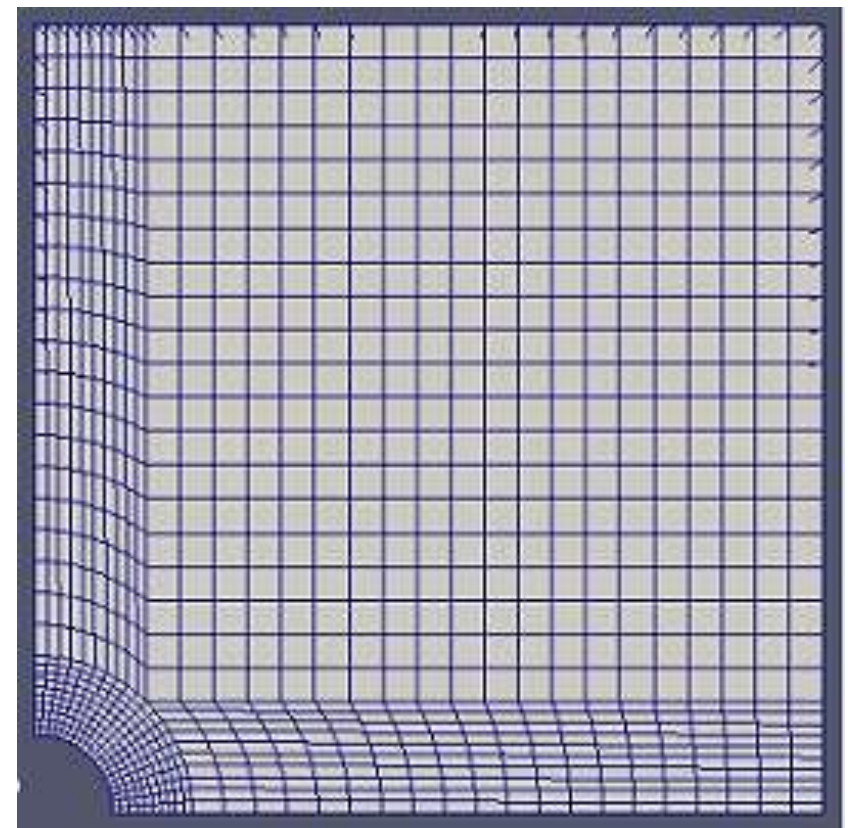

Fig-5:Full view of the finite volume grid for $\mathrm{L} / \mathrm{d}=5$. 
The alternative Gauss Seidel (GS) has been adopted to solve velocity with residual criteria of eps $=1 \mathrm{e}-5$ and under relaxation factor of $\alpha_{u}=\alpha_{u}=0.6$, to solve the pressure equation, the values of eps $=1 \mathrm{e}-5$ and $\alpha_{p}=0.1$ are used.

Fig 6 shows the location of yield surfaces at $\mathrm{Od}=10,100$, 1000 and 10000. The comparison of numerical and result of Tokpavi et al.[13] shows good agreement, a minor difference was observed may be due to use of coarse mesh.
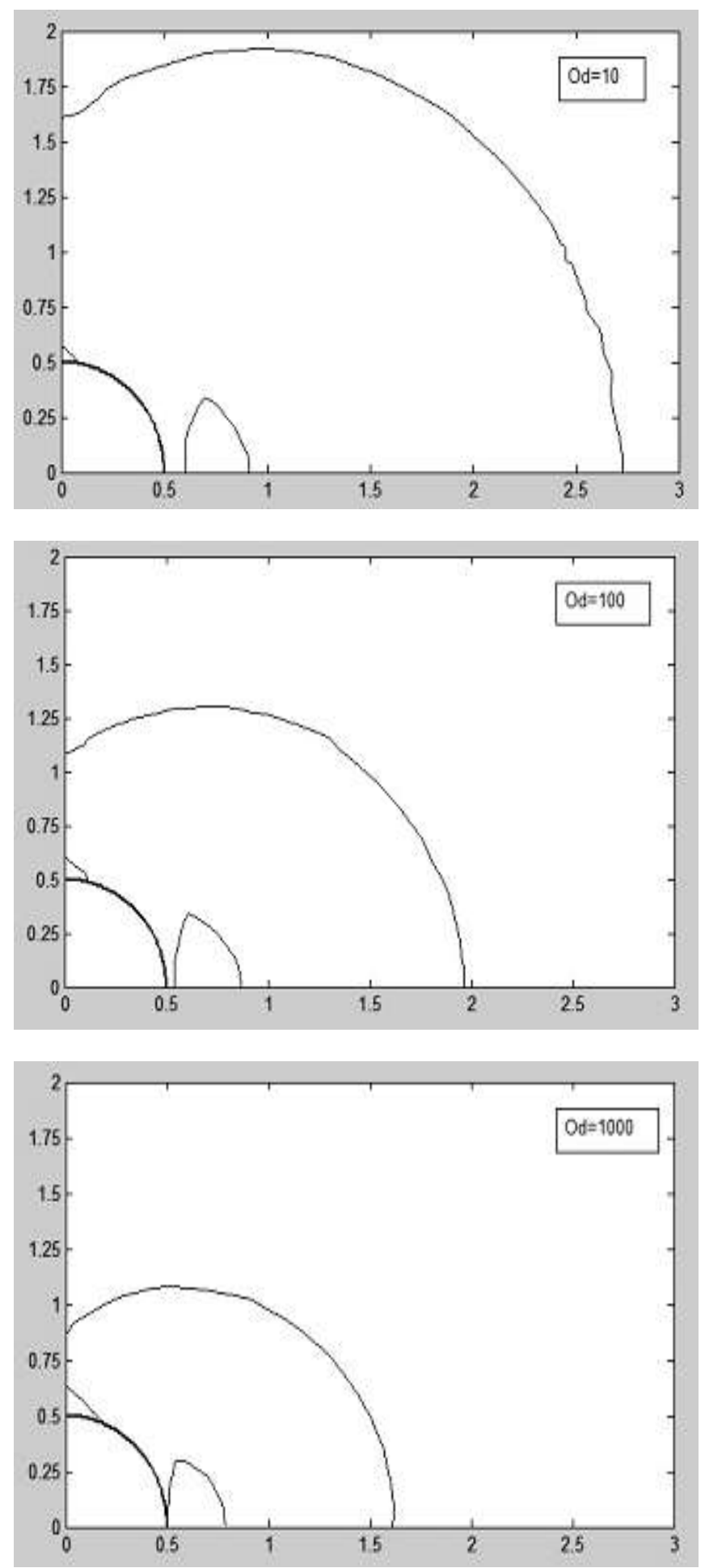

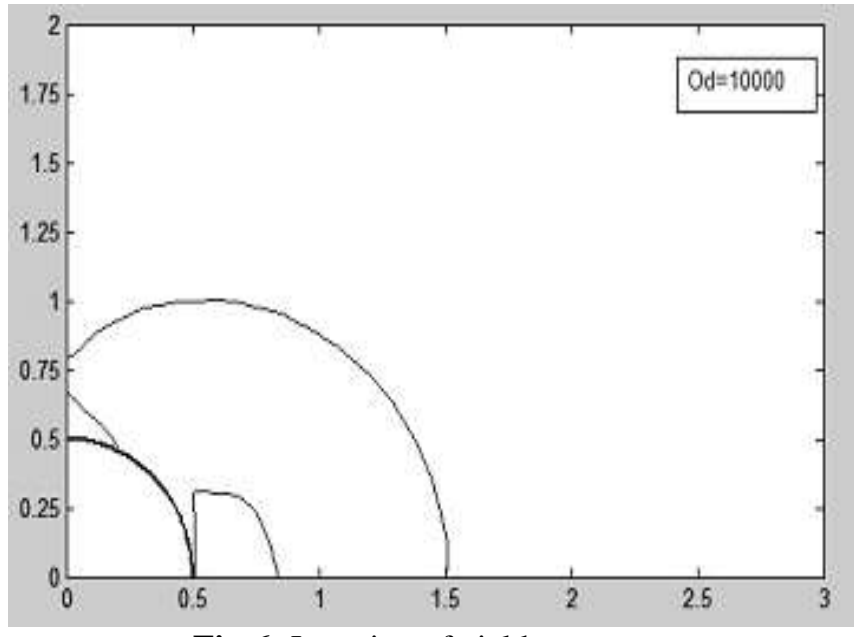

Fig-6: Location of yield structure

Fig 7 shows the changes in yield surfaces $(\mathrm{S} 1, \mathrm{~S} 2, \mathrm{~S} 3)$ for Oldroyd number of 10, 100, 1000, 10000.

As can be seen from Fig 6 and Fig 7 the space of the rigid zone increased by increasing the Oldroyd number.
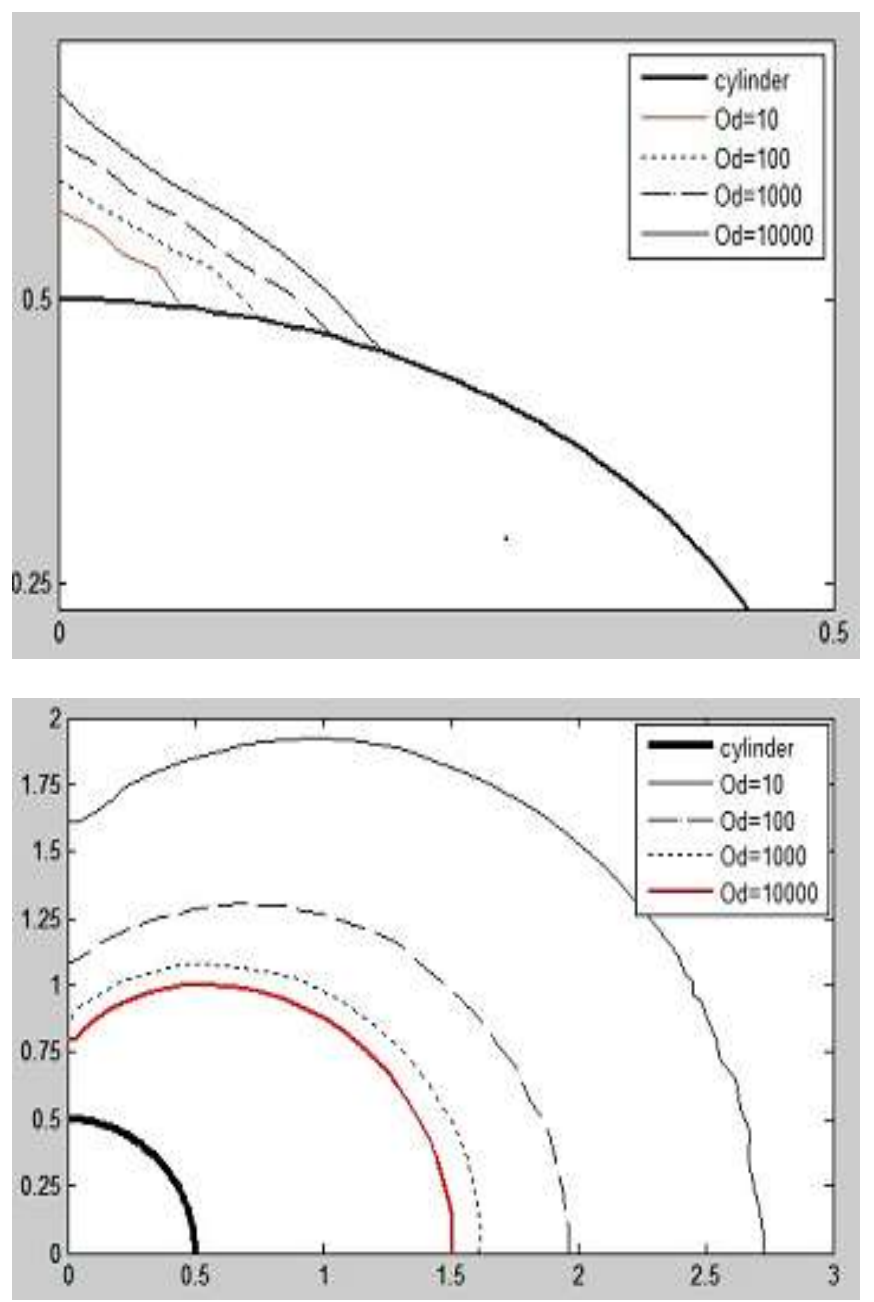


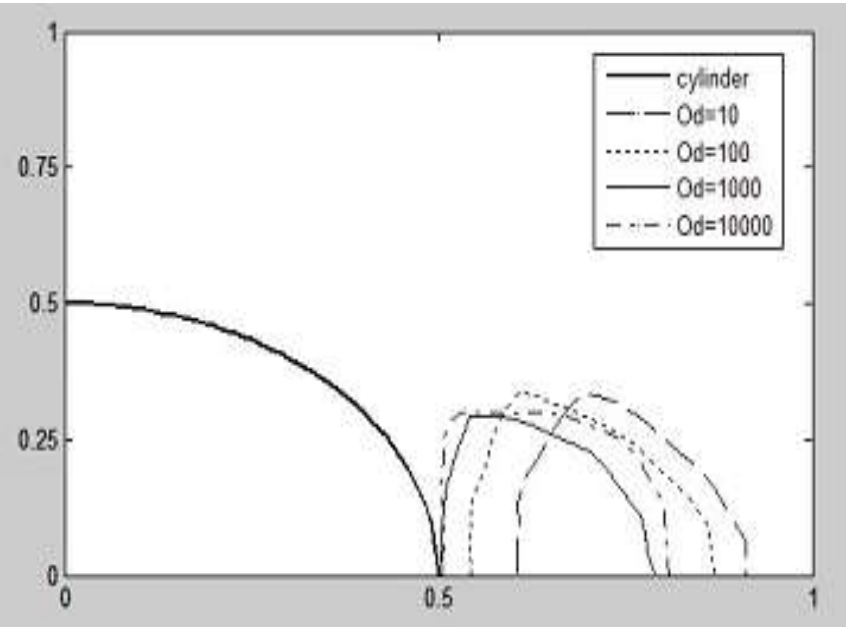

Fig-7: Dependence of yield surfaces S1, S2, S3 on Oldroyd number

Increasing at large Oldroyd number as created slightly change in rigid zones.

\section{CONCLUSION}

In this paper a Finite Volume Method has been implemented for the creeping flow past a circular cylinder. The SYMPLE algorithm adopted the equation discretized on a staggered grid. The location of yield surfaces identified over the range of Oldroyd number of 10,100,1000, 10000. Results are compared with obtained results from finite element method, and shows good agreement. The present model could be useful for solving creeping flow problem and could be a good alternative to the other solver like finite element and commercial COMSOL software.

\section{REFERENCES}

[1]. Adachi, K. N.Yoshioka, On creeping flow of aviscoplastic fluid ast a circular cylinder. Chemical Engineering Science. 28(1973) 215-226.

[2]. Baaijens, H.P.W., Peters, G.W.M., Baaijens, F.P.T., Meijer, H.E.H., Viscoelastic flow past a confined cylinder of a polyisobutylene solution, J. Rheol. 39 (1995) 1243-1277. [3]. Beris, A.N., Tsamopoulos, J.A., Armstrong, R.C., Brown, R.A., Creeping motion of a sphere through a Bingham plastic, J. Fluid Mech. 158 (1985) 219-244.

[3]. Bird, R.B., Dai, G.C., Yarusso, B.J., The rheology and flow of viscoplastic materials, Rev. Chem. Eng. 1 (1983) 170 .

[4]. Keei, D.De, Turcotte, G., Viscosity of biomaterials. Cheml Eng. 6(1980) 273-282.

[5]. Liu, A.W., Bornside, D.E., Armstrong, R.C., Brown, R.A. , Viscoelastic flow of polymer solutions around a periodic, linear array of cylinders: comparisons of predictions for microstructure and flow fields, J. NonNewtonian Fluid Mech. 77 (1998) 153-190.

[6]. Mitsoulis, E., Flows of Viscoplastic materials: Models and Computations, Rheology Reviews (2007), 135 - 178.

[7]. Mitsoulis, E., Abdali, S., Flow simulations of HerschelBulkley fluid through extrusion dies,Can.J. Chem. Eng.71 (1993) 147-160.
[8]. Mitsoulis. Evan, On creeping drag flow of a viscoplastic fluid past a circular cylinder: wall effects, Chemical Engineering Science 59 (2004) 789-800.

[9]. Mallick, Monalisa, Kumar, A., Study on Drag Coefficient for the Flow Past a Cylinder, International Journal of Civil Engineering Research. 2278-3652 (2014), 301-306.

[10]. Nirmalkar, N., Chhabra, R.P., Poole, R.J., On creeping flow of a Bingham plastic fluid past a square cylinder,Journal of Non-Newtonian Fluid Mechanics 171172 (2012) 17-30.

[11]. Papanastasiou, T.C.,Flow of materials with yield, J. Rheol. 31 (1987) 385-404.

[12]. Roquet, N., Saramito, P., An adaptive finite element method for Bingham fluid flows around a cylinder, Comput. Meth. Appl. Mech. Eng. 192 (2003) 3317-3341.

[13]. Tokpavi, D.L., Magnin, A., Jay, P., Very slow flow of Bingham viscoplastic flow around a circular cylinder, J. Non-Newton. Fluid Mech. 154 (2008) 65-76.

[14]. Zhu, H., Kim, Y.D., Kee, D.De, Non-Newtonian fluids with a yield stress. J. Non-Newtonian Fluid Mech. 129 (2005) 177-181.

[15]. Zisis, Th., Mitsoulis, E., Viscoplastic flow around a cylinder kept between parallel plates. J. Non-Newtonian Fluid Mech. 105 (2002) 1-20.

\section{BIOGRAPHIES}

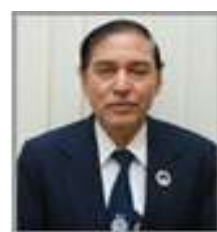

Professor and head in the Mathematics Department Mathematics Institute of Technology, Pune, Maharashtra, India

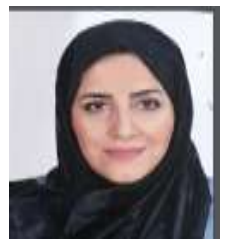

Phd Student at Department of Mathematics, S. P. Pune University, Pune, Maharashtra, India 